\title{
Polimorfismo de citoquinas relacionadas ao processo inflamatório periodontal
}

\author{
Fabiana Cervo de Barros ${ }^{1}$ \\ Carlos M arcelo S. Figueredo ${ }^{2}$ \\ Ricardo G. Fischer ${ }^{3}$
}

\begin{abstract}
Resumo
0 objetivo destetrabal ho foi fazer uma revisão da literatura a respeito do polimorfismo em citoquinas relacionadas ao processo inflamatório periodontal ede como esse fator genético poderia estar ligado ao desencadeamento da doença destrutiva do periodonto. Foram levados em consideração estudos sobre determinadas citoquinas, como: fator de necrosetumoral- alfa (TN F $\alpha$ ), interleucina-1-alfa (IL-1 $\alpha$ ), I L-1 beta (IL-1 $\beta$ ) ou IL-1 receptor antagonista (I L-IRA), IL-2, IL-4 , I - 6, IL-10, I L-16 el L-18. A revisão da literatura possibilitou concluirmosque: (1) em al gumas citoquinas, como IL-1, IL-2, IL-4, IL-6, IL-10, há variação nos resultados em relação à presença do gene polimórfico e 0 desenvolvimento da periodontite; (2) algunsgenes polimórficos, como os da IL-16 el L-18, não apresentam relação com a periodontite; (3) existe um enfoque relevante em relação aos gruposétnicos eraciais; (4) o genótipo para IL-1B está associado ao risco para periodontite severa, na população diabética, mas os resultados são contraditórios quando 0 consumo de cigarros éestudado.
\end{abstract}

Palavras - chave: polimorfismo - citoquinas- doença periodontal; periodontite- fumo;periodontite- diabetes mellitus.

\section{INT RO DUÇÃO}

A doença periodontal (DP) é formada por um grupo de doenças que afeta a gengiva e as estruturas de suporte dos dentes. $\mathrm{Na}$ gengivite, observa-se, clinicamente, edema, vermelhidão, sangramento à sondagem com alteração no contorno e na consistência da gengiva; na periodontite, há destruição dos tecidos de suporte do dente (cemento radicular, ligamento periodontal e osso alveolar), observada clinicamente por perda de inserção e formação de bolsa periodontal (KIN ANE; HART, 2003).

Fatores microbiológicos e ambientais são ditos como responsáveis pelo início e modula- ção da doença periodontal, e a variável bacteriana parece não explicar a maioria dos progressos entre gengivite e periodontite (KORN M AN et al., 1997). Existem fortes evidências que suportam que a expressão genética influencia na predisposição e progressão da doença periodontal (HART, 1994; MICHALOWICZ, 1994; H ASSELL; H ARRIS, 1995; H ART, 1996) eque os processos de destruiç̧ão, na periodontite, são derivados do indivíduo (N ARES, 2003). 0 resultado e a progressão da DP constituem um processo que depende da interação de várias citoquinas pró ou antiinfla-matórias de efeitos

\footnotetext{
${ }^{1}$ M estranda do Programa de Pós-graduação em Periodontia. UERJ. Rio de Janeiro - RJ

${ }^{3}$ Professor Titular de Periodontia. UERJ. Rio de Janeiro - RJ

\section{Correspondência para/ Correspondenceto:}

Universidade do Estado do Rio de Janeiro -Faculdade de O dontologia

A/C Fabiana C. de Barros Barroso

Av. Boulevard 28 de Setembro, no 157 - Vila Izabel

20551-030 Rio de Janeiro- RJ - Brasil

Tel.: (21) 2587-6313; (21) 9959-7743.

E-mail: fabianacbarros@bol.com.br
}

2 Professor Adjunto de Periodontia. PU C-RJ, UNIGRAN RIO e UERJ. Rio de Janeiro - RJ 
sinérgicos e antagônicos (TREVILAT TO et al., 2003). Possivelmente, os níveis de secreção das citoquinas que estimulam células afetam o metabolismo ósseo e a degradação da matriz extracelular (LANDI et al., 1997; CULLIN AN et al., 2001) e podem ser alterados por uma variação genética. Assim, o polimorfismo gênico tem sido considerado como instrumento importante para identificar indivíduos susceptíveis a doenças com patogênese inflamatória (DUFF, 1998; SOGA et al., 2003). 0 risco genético para o desenvolvimento da periodontite vem sendo estudado em populações distintas, uma vez que forte relação com os grupos étnicos eraciais pode ser traçada com o polimorfismo das citoquinas estudadas (PAPAPAN OU et al., 2001; M OREIRA et al., 2005). Além disso, a possibilidade de associação do fator de risco ge nético com outros fatores de risco, como fumo e diabetes mellitus, é levantada por alguns trabalhos (PARKHILL et al., 2000; GUZMAN et al., 2003; M EISEL et al., 2003).

Trabalhos iniciais como os de Kornman e colaboradores (1997) e de Gore e colaboradores (1998) associam um genótipo específico para a produção de IL-1 1 (+3954) e IL-1 $\alpha$ (889) com o aumento na produção de IL-1, indicando que isso seria um forte marcador para a susceptibilidade à periodontite do adulto. No entanto, trabalhos subseqüentes podem não observar tal relação e ainda envolvem, em suas pesquisas, outras citoquinas também presentes no processo inflamatório (YAMAZAKI et al., 2001; AN USAKSATHIEN et al., 2003; MOREIRA et al., 2005; TREVILATTO et al., 2003; G O N ZALES et al., 2004; FO LWACZNY et al., 2005b).

0 objetivo deste trabalho foi fazer uma revisão da literatura a respeito do polimorfismo em citoquinas relacionadas ao processo inflamatório periodontal e como esse fator genético poderia estar ligado ao desencadeamento da doença destrutiva do periodonto.

\section{REVISÃO DE LITERATURA}

\section{Citoquinas no processo inflamatório}

As citoquinas são proteínas regulatórias envolvidas no início e na progressão da doença inflamatória crônica (KO R N M AN et al., 1997; PAPAPAN OU et al., 2001). Elas podem ter funções pró ou antiinflamatórias (TREVILATTO et al., 2003). A desregulação da expressão genética das citoquinas pode ser responsável pelos repetidos ciclos de inflamação tecidual observados na DP (DUFF, 1998). Certas citoquinas têm seus genes estudados quanto à presença de polimorfismos em sua codificação genética, tais como: fator de necrose tumoralalfa (TNF $\alpha$ ). interleuquina (IL) IL-1 alfa $(\alpha)$, IL-1 beta $(\beta)$ e IL-1 receptor antagonista (RA), IL-2, IL-4, IL- 6, IL-10, IL-16 e IL-18. 0 genótipo para algumas citoquinas influencia diretamente na patogênese da $D P$ pelo efeito na síntese dessas citoquinas (M IC H ALOW ICZ, 1994; H ART; KO RN M AN, 1997; PARKH ILL et al., 2000). Para estudarmos o polimorfismo nas citoquinas, torna-se importante saber quais são as funções de tais citoquinas.

Acredita-se que a IL-1 seja a citoquina crucial na patogênese da DP (MEISEL et al., 2003). Ela é a primeira ativadora da quimiotaxia de outras citoquinas, bem como da expressão de moléculas de adesão, que facilitam a migração dos leucócitos dentro dos tecidos (LAN G et al., 2000; CULLINAN et al., 2001). Existem três genes que regulam a produção de IL1: IL-1A, IL-1B , que controlam a produção de proteínas pró- inflamatórias IL-1 $\alpha$ e IL-1 $\beta$, respectivamente, e o gene IL-1 RN, que controla a síntese da proteína antagonista à IL-1 (ILIRA) (GREEN STEIN; HART, 1997). A IL-1 $\alpha$ e $\beta$ e o T N F $\alpha$ são produzidos por céluIas Th 1 (JANEWAY et al., 2000), por monócitos/macrófagos, durante a resposta inflamatória inicial (SO G A et al., 2003), e estimulam muitas células a produzirem metaloproteinases de matriz, prostaglandinas (PG) e citoquinas pró-inflamatórias, bem como afetam o metabolismo ósseo, o que contribui para a patogênese da DP (LANDI et al.,1997; CULLIN AN et al.,2001). A função da IL-1 RA é de se ligar à IL-1, bloqueando-a, e assim pre venindo a ativação das células-alvo (DINARELLO , 1996).

A IL-2 é uma citoquina produzida pela célulaT helper 1 (Th1) (JAN EWAY et al., 2000; SCAREL - CAMINAGA et al., 2002; GON - 
ZALES et al., 2004) e está envolvida na ativação da célula B, na estimulação de macrófagos e células natural Killer, na proliferação de células T e na atividade osteoclástica que ocorre durante a reabsorção óssea (SCAREL - CAM IN AGA et al., 2002).

A IL-4 é produzida por células $T$ helper 2 (Th2) (PAUL, 1991; HAJEER et al., 1998) e sua função é ativar essas células, regular imunoglobulinas, estimular a proliferação de células B (PAUL, 1991), inibindo a secreção de prostaglandina E2 e citoquinas feitas pelos macrófagos (TE VELDE et al., 1990 ; CO RC O RAN et al., 1992).

Assim como as citoquinas já citadas, a IL6 também apresenta múltiplas funções, como participar da diferenciação da célula B e da proliferação das células $T$, estimular a hematopoiese e acelerar a reabsorção óssea (BO CH ; WARAASWAPATI; AU RON, 2001). Regula a resposta imunológica, e seus efeitos se sobrepõem aos da IL-1 e do TNF (FUJIHASHI et al., 1993). Além disso, é produto das células Th2 (JANEWAY et al., 2000).

IL-10 é uma citoquina antiinflamatória, produzida por células T Helper 2 (H AJEER et al., 1998; J AN EW AY et al ., 2000) e macrófagos, que inibe a síntese de citoquinas pró-inflamatórias, como a IL-1, IL6, IL-8, (H AJEER et al., 1998) e também IL-2, TN $F \alpha$ e IN $F \gamma$, as quais são produzidas por células T H elper 1(LALAN I; BHOL; AHMED, 1997; JANEWAY et al., 2000). Além disso, estimula a proliferação de células $B$ e a produção de anticorpos (LALAN I; BH O L; AH M ED, 1997; H AJEER et al., 1998).

A IL-16 vem sendo estudada quanto ao seu envolvimento com doenças crônicas inflamatórias (LABERGE et al., 1997; SCH REIBER, 2001). Sua função é ativar células T, monócitos, macrófagos e células dendríticas, ligando-se à molécula CD 4 e estimulando a produção dessa citoquina. A ativação das células $\mathrm{T}$ estimula a produção de diferentes citoquinas, como TN $F$ $\alpha$, IL-1 $\beta$ IL-18 por monócitos. (M ATH Y et al., 2000).

Por sua vez, a IL-18 é produzida por macrófagos, monócitos eosteoblastos (GERDES et al., 2002), está envolvida na regulação do sistema inato e adquirido de defesa (DIN ARE-
LLO ; FANTUZZI, 2003), estimula a expressão de TNF $\alpha$ e IL1, a diferenciação de células T em Th1, induz a produção de IN F $\gamma$ na pre sença de IL-12 e inibe a síntese de citoquina anti-inflamatória IL-10 (FO LWA-CZNY et al., 2005a).

\section{Polimorfismo nas citoquinas envolvidas na periodontite}

Polimorfismo é uma variação da seqüência de nucleotídeos em um alelo de um gene, que pode ocorrer por transcrição ou substituição (KIN AN E; H ART, 2003; TREVILATTO et al., 2003). Essa variação pode acontecer em uma região do exon ou do intron do DNA, ou do RNA, que será usado para a produção de proteínas (N ARES, 2003). Q uando um alelo específico ocorre em uma freqüência relativamente elevada na população $(>1 \%)$, diz-se que ele apresenta um polimorfismo genético (KINANE; HART, 2003; GUZMAN et al., 2003; N ARES, 2003). Q uando essa alteração no nucleotídeo é muito rara, e não presente em muitos indivíduos, isso é freqüentemente chamado de mutação (KIN AN E; H ART, 2003; NARES, 2003). 0 gene pode apresentar polimorfismo em somente um alelo, ou até em 5 alelos (PARKH ILL et al., 2000; TAl et al., 2002; AN U SAKSATHIEN et al., 2003), e 0 genótipo positivo é definido como a presença de pelo menos um alelo raro em cada locus (PAPAPAN OU et al., 2001).

Certos polimorfismos podem aumentar ou diminuir o risco de a pessoa desenvolver 0 fenótipo para a doença (KINANE; HART, 2003). 0 estudo dessas variações genéticas nas citoquinas pode contribuir para entender a interação dos mediadores do hospedeiro que determinam o fenótipo da doença (TREVILATTO et al., 2003). Cada variante, usualmente, não causa doença por si só, mas é possível que uma combinação específica de polimorfismos em genes diferentes ou a interação com fatores ambientais possam alterar significan-temente o risco de o indivíduo desenvolver 0 fenótipo para certas doenças (GUZM AN et al., 2003; G O N ZALES et al., 2004). G enótipos particulares de citoquinas influenciam diretamente na patogênese da doença, pois afetam a 
síntese das citoquinas (MICHALOWICZ, 1994; HART; KORN-MAN, 1997; PARKHILL et al., 2000). 0 utros autores, no entanto, não conseguem achar relação entre a presença do polimorfismo e o desenvolvimento da doença (GONZALES et al., 2004; FO LWACZN Y et al., 2005a; FO LW-ACZN Y et al., 2005b). Algumas citoquinas pró-inflamatórias apresentam um possível envolvi-mento com o polimorfismo genético. São elas T N F $\alpha$, IL-1 $\alpha$ IL- $1 \beta$, IL-1 RA (VNTR), IL-2, IL-4 ,IL-6, IL-10, IL-16 e IL-18, sendo as mais estudadas a IL1 e a IL10 (KIN ANE; H ART, 2003; TREVILATTO et al., 2003; FO LWACZNY et al., 2005a; FOLWACZNY et al., 2005b). Para cada uma dessas substâncias, existe uma ou mais regiões do gene (locus) identificadas como responsáveis pela variação alélica; tais citoquinas e suas respectivas variações podem ser vistas no Q uadro 1 .

\section{Inter-relação entre polimorfismo, periodontites crônica e agressiva e os grupos étnico-raciais.}

0 polimorfismo genético pode ser associado com diferentes formas clínicas de periodontite (KORNMAN et al., 1997). A periodontite agressiva (PA) sofre forte influência dos fatores genéticos (H ART, 1996). No que se refere à periodontite crônica, certos estudos afirmam que ela é aparentemente pouco influenciada por esses fatores (PARK HILL et al., 2000; TAl et al., 2002). No entanto, tem-se demonstrado que a susceptibilidade à periodontite crônica (PC) também pode estar

\begin{tabular}{|c|c|c|c|c|c|c|c|}
\hline Citoquinas & \multicolumn{7}{|c|}{ Regiós de polimorfismo } \\
\hline $\mathrm{IL}-1 \alpha$ & -889 & +4845 & & & & & $\begin{array}{l}\text { Cullinan et al., 2001; } \\
\text { Kinane; Hart, 2003; } \\
\text { Gonzáles et al., } 2003\end{array}$ \\
\hline IL-1 $\beta$ & -511 & $\begin{array}{c}+3953 \\
\text { ou } \\
+3954\end{array}$ & -31 & & & & $\begin{array}{l}\text { Cullinan et al., 2001; } \\
\text { Parkhill et al., 2000; } \\
\text { Anusaksathien et al., 2003; } \\
\text { Kinane; Hart, 2003; Soga et } \\
\text { al., 2003; Guzman et al., } \\
\text { 2003; Moreira et al., 2005? }\end{array}$ \\
\hline IL1RA (VNTR) & +2018 & Intron 2 & & & & & $\begin{array}{l}\text { Parkhill et al., 2000; Tai et } \\
\text { al., 2002; Guzman et al., } \\
2003\end{array}$ \\
\hline TNF $\alpha$ & -1013 & -863 & -857 & -308 & -238 & +252 & $\begin{array}{l}\text { Kinane; Hart, 2003; Soga } \\
\text { et al., } 2003\end{array}$ \\
\hline IL-2 & -330 & & & & & & Scarel- Caminaga et al., 2002 \\
\hline IL-4 & -590 & Intron 2 & & & & & $\begin{array}{l}\text { Michel et al., } 2001 ; \\
\text { Gonzales et al., } 2004\end{array}$ \\
\hline IL-6 & -597 & -572 & -174 & & & & $\begin{array}{l}\text { Trevilla to et al., 2003; } \\
\text { Holla et } \\
\text { al., } 2004\end{array}$ \\
\hline $\mathrm{IL}-10$ & -1140 & $\begin{array}{c}-1082 \\
\text { ou } \\
-1087\end{array}$ & $\begin{array}{l}-824 \\
\text { ou } \\
-819\end{array}$ & $\begin{array}{l}-592 \\
\text { ou } \\
-597\end{array}$ & -506 & & $\begin{array}{l}\text { Yamazaki et al., 2001; } \\
\text { Gonzales et al., 2002; } \\
\text { Berglundh et al., 2003; } \\
\text { Scarel- Caminaga et al.,2004 }\end{array}$ \\
\hline IL-16 & -295 & & & & & & Folwaczny et al., 2005b \\
\hline IL-18 & -656 & -607 & -137 & +113 & +127 & +105 & Folwaczny et al., 2005a \\
\hline
\end{tabular}

Q uadro 1- Citoquinas eas regiões de polimorfismo estudadas 
relacionada com o componente hereditário em $50 \%$ dos casos (KORNMAN et al., 1997; MICH ALOWICZ et al., 2000).

A presença do alelo 2 no polimorfismo para IL-1 é um fator de risco para periodontite severa em caucasianos (KORN MAN et al., 1997) e também em brasileiros (M O REIRA et al., 2005). Q uando comparada à incidência do alelo 1 ou 2 para IL-1 B (+3954), nas formas agressiva e crônica da periodontite, uma diferença na patogênese dessas formas clínicas etambém nos grupos étnicos e raciais pode ser vista entre caucasianos e japoneses (TAl et al., 2002); quanto à clínica, nenhuma associação entre 0 gene para IL-1 13 (+3954) e a PA foi encontrada (TAl et al., 2002; MOREIRA et al., 2005); enquanto que o polimorfismo para IL-1 $\beta$ (+3954) influencia na severidade da DP em caucasianos, em japoneses isso não ocorre (SO GA et al., 2003). 0 alelo 2 para IL-1 B (+3954), por sua vez, tem sua freqüência estatisticamente aumentada entre os pacientes caucasianos com periodontite severa avançada e indivíduos com doença média ou moderada (GORE et al., 1998). Um aumento da severidade da perda de inserção nos pacientes com genótipo positivo para IL-1 pode ser observado.(PAPAPAN OU et al., 2001). Todavia, a distribuição do genótipo positivo foi tão pequena, que não foi possível mensurar a relação entre a composição genotípica da IL-1 $\beta$ (+3954) e IL-1 $\alpha$ (-889) e a periodontite agressiva ou crônica nas populações taiandesa ou chinesa (AN U SAKSATHIEN et al., 2003). 0 carreamento do alelo 2 para IL-1 a (+4845), IL-1 $(+3954)$ e IL-1RN é menos incidente em japoneses que em caucasianos; já para IL-1 $\beta$ (-511), é semelhante entre japoneses e chineses (TAl et al., 2002). 0 carreamento do alelo 2 para IL-1RN não está associado à severidade da DP (KORNMAN et al., 1997; PARKHILL et al., 2000); no entanto, para TAl et al. (2002), a freqüência de polimorfismos nos alelos 2, 3, 4, e 5 desse gene está aumentada em pacientes com PA generalizada. Em contraste, homozigotos para 0 alelo 1 da IL-1 $\beta$ (+3954) têm sua freqüência para PA localizada ou generalizada aumentada em caucasianos, quando comparada ao controle, talvez pelo efeito na expressão desse alelo (PARKH ILL et al., 2000). A transmissão do alelo 1 para IL-1A (-889) é mais freqüente que 0 alelo $2 \mathrm{em}$ pacientes com periodontite agressiva na população caucasiana. GUZMAN et al. (2003) e GONZALES et al. (2003) não observaram associação alguma entre polimorfismo para IL-1 A ou B, e a doença periodontal agressiva nas populações de caucasianos norte-europeus ou hispânicos da América Central. 0 genótipo contribui, mas não é fator de risco essencial para o desenvolvimento da DP em adultos australianos caucasianos (CULLIN AN et al ., 2001).

No que diz respeito ao TN F $\alpha(-1013)$ 863 e -857) e à IL-2 (-330), há associação entre esses polimorfismos e a severidade da D P nas populações japonesa e brasileira, respectivamente (SCAREL- CAM IN AGA et al 2002; SO GA et al., 2003).

Em pacientes com $\mathrm{PA}$, caucasianos $\mathrm{N}$ orte-europeus na maioria, os dados mostram interação positiva entre o polimorfismo para IL4 no promoter e intron $(P P+$ e IP+) e a DP (M ICHEL et al., 2001). Por outro lado, a mesma interação não foi vista em japoneses caucasianos com PA (G O N ZALES et al., 2004), nem em coreanos com periodontite do adulto (KAN G et al., 2003), ou no grupo de brasileiros de origem africana com DP (PONTES et al., 2004). Sendo assim, talvez a IL-4, por si mesma, não possa ser considerada um fator biológico que contribui para a etiopatogenia da PA (GONZALES et al., 2004).

U ma relação positiva entre a presença do polimorfismo para IL-6 (-174) e a susceptibilidade à DP crônica em caucasianos brasileiros é confirmada pelo aumento na freqüência do genótipo $G / G$ nessa região promotora do gene (TREVILATTO et al., 2003). No entanto, H olla e colaboradores (2004) não observaram relação entre esse polimorfismo e a periodontite. Talvez, o polimorfismo para IL-6 (-572) possa ser relevante como fator protetor, associado à baixa susceptibilidade à periodontite do adulto (H O LLA et al., 2004).

O s polimorfismos na IL-10 (-1082/ -819 e-592) não parecem ser fortes determinantes para susceptibilidade em desenvolver periodontite crônica ou agressiva em indivíduos japone- 
ses (YAM AZAKI et al., 2001; GON ZALES et al., 2002). N o entanto, alguma relação da IL10 (-1087) em caucasianos do norte europeu pode estar associada à PC severa, sendo que a presença do alelo $G$, na posição -1087 , estaria relacionada com 0 aumento na produção da IL10, enquanto o alelo A, nessa posição, levaria a um decréscimo na produção dessa proteína (BERGLUNDH et al., 2003). O utros resultados ainda mostram que há relação entre os polimorfismos IL-10 (-819 e -592) e a susceptibilidade para PC, mas não com a IL-10 (1087) em caucasianos brasileiros (SCAREL CAM IN AGA et al., 2004).

$N$ enhuma associação foi encontrada entre quaisquer dos polimorfismos para IL-16 (295) ou IL-18 (-656/ -607/ -137/ +113/ +127/ +105) em caucasianos e a susceptibilidade à periodontite (FO LWACZNY et al., 2005a; FOLWACZNY et al., 2005b).

A freqüência de muitos alelos varia entre os grupos étnicos, e muitos estudos têm mostrado resultados contraditórios quando são feitas comparações entre populações distintas (PAPAPAN OU et al., 2001; SOGA et al., 2003; M OREIRA et al., 2005). Múltiplos genes podem influenciar a susceptibilidade ou severidade à doença no indivíduo (SO G A et al., 2003). A presença do gene polimórfico pode aumentar ou diminuir o risco de o indivíduo desenvolver o fenótipo para doença (KINANE; HART, 2003). O genótipo para diversas citoquinas pode influenciar diretamente na patogênese da $D P$, pois afeta a síntese das citoquinas (M ICH ALOWICZ, 1994; HART; KORN M AN, 1997; PARKHILL et al., 2000). No entanto, os autores não são unânimes em suportar uma relação positiva entre a composição genotípica e a susceptibilidade ou severidade à doença periodontal (GONZALES et al., 2004; FOLWACZNY et al., 2005a; FO LW ACZNY et al., 2005b). Talvez isso ocorra porque muitos dos polimorfismos podem depender da presença de outros genes polimórficos ou de fatores ambientais para manifestar uma contribuição no risco para o desenvolvimento da doença (GO N ZALES et al., 2004).

\section{Evidências com os fatores de risco}

As evidências com os fatores de risco são levantadas em relação a I L-1 e o diabetes mellitus etambém dessa citoquina com o fumo. Enquanto os genótipos IL-1A (+ 4845) e IL-1RN (+2018) não mostram relação com a periodontite, os genótipos IL-1B (-511) e IL-1 B (+3954) apresentam possível associação com a DP destrutiva; assim, o risco de desenvolvimento de periodontite severa na população diabética relaciona-se ao polimorfismo na IL-1 B (GUZM AN et al., 2003). Alguns autores sugerem que o fumo e o genótipo para IL-1 são fatores independentes na periodontite severa, e que a presença de pacientes fumantes na amostra poderia ocultar o risco ao polimorfismo das citoquinas (KORNMAN et al., 1997; PAPAPANOU et al., 2001; MOREIRA et al., 2005). Existem relatos de associação com 0 genótipo 1/1 na IL-1B (+3954) em pacientes fumantes com periodontite de início precoce (PARKH ILL et al., 2000) e também uma forte relação pode ser encontrada do genótipo positivo para IL-1 em pacientes com a forma severa da periodontite em fumantes, sugerindo um sinergismo entre o fumo e o fator de risco genético para IL-1 (M EISEL et al., 2003). Por outro lado, nenhuma associação significante foi vista entre fumo e IL-1A (+4845) ou IL-1B (+3954), mas sim na freqüência aumentada de genes polimórficos para IL-1 RN (VNTR) em pacientes não fumantes com periodontite de início precoce generalizada (TAl et al., 2002) e na presença de aumento na profundidade de bolsa em pacientes não fumantes com genótipo positivo para IL-1(CULLINAN et al., 2001). A determinação da associação entre fumo e a severidade da doença periodontal em pacientes com polimorfismo para IL-1 ou outras citoquinas pode ser dificultada pelo fato de a amostra de fumante ser pequena (GON ZALES et al., 2003), ou pela não inclusão desses pacientes nos estudos (TREVILAT TO et al., 2003; SCAREL - CAM IN AGA et al., 2004), ou ainda por não haver relato da inclusão ou exclusão desse fator de risco (YAM AZAKI et al., 2001; M ICH EL et al., 2001; SCAREL - CAM IN AGA et al., 2002; GONZALES et al., 2002; 
BERGLUNDH et al., 2003; SOGA et al., 2003; G ON ZALES et al., 2004; FO LWACZNY et al., 2005a; FOLWACZNY et al., 2005b).

\section{CONCLUSÃO}

O s trabal hos sobre polimorfismo genético, nas diversas citoquinas relacionadas com 0 processo inflamatório periodontal, mostram que:

1 - Em algumas citoquinas, como IL-1, IL-2, IL-4, IL-6, IL-10, há variação nos resul- tados em relação à presença do gene polimórfico e o desenvolvimento da periodontite.

2 - Alguns genes polimórficos, como os da IL-16 e IL-18, não apresentam relação com a periodontite;

3 - Existe um enfoque relevante em relação aos grupos étnicos e raciais.

4 - 0 genótipo para IL-1B está associado ao risco para periodontite severa na população diabética, mas os resultados são contraditórios quando o consumo de cigarros é estudado.

\title{
Polymorphism in cytokinesassociated with the periodontal inflammatory process
}

\begin{abstract}
The aim of this study was to make a review of literature about polymorphism in cytokine associated with the periodontal inflammatory process and how this genetic factor may be associated with development of periodontal destructive disease. Studies about some citokines include: tumor necrosis factor - alpha (TNF- $\alpha$ ), interleukin -1 - alpha (IL-1 $\alpha$ ), beta (IL-1 $1 \beta$ ) ou IL-1 antagonist receptor (IL-1RA), IL-2, IL-4 ,IL- 6, IL-10, IL-16 e IL-18. This review make possible conclusions that: 1- in some citokines like: $\mathrm{IL}-1, \mathrm{IL}-2, \mathrm{IL}-4, \mathrm{IL}-6, \mathrm{IL}-10$, the results have variation in relationship of polymorphic gene and periodontitis development; 2- some polymorphic genes, as IL-16 e IL-18 do not have relation with periodontitis; 3- there is a relevant emphasis of the relationship with ethnic and racial groups; 4- the genotype for IL-1B is associated with the risk for severe periodontitis on diabetic population, but the results are conflicted when smoking is considered..
\end{abstract}

Key words. Polymorphism, interleukin, periodontal disease, smoking, diabetes mellitus

\section{REFERÊNCIAS}

AN U SAKSATHIEN, 0 , et al. Distribution of interleukin-1beta(+3954) and IL-1alpha(-889) genetic variations in a Thai population group. J. Periodontol., Chicago, v.74, n.12, p.17961802, Dec. 2003.

BERGLUNDH, T. et al. Association of the 1087 IL 10 gene polymorphism with severe chronic periodontitis in Swedish Caucasians.

J. Clin. Periodontol., Copenhagen, v.30, n.3, p.249-254, M ar. 2003.

BOCH , J.A.; WARA-ASWAPATI, N .; AU RO N , P.E. IL-1 signal transduction- current concepts and relevance to periodontitis. J. D ent. Res., Alexandria, v.80, n.2, p.400-407, Feb. 2001.

CORCORAN, M.L. et al. IL-4 inhibition of PGE2 synthesis block interstitial coollagenase and $92 \mathrm{~K}$ d type IV collagenase/ gelatinase production by human monocytes. J. Biol. Chem., Baltimore, v.267, p.515-519, 1992.

CULLINAN, M.P. et al. A longitudinal study of interleukin-1 gene polymorphisms and periodontal disease in a general adult popu- 
Iation. J. C lin. Periodontol., C openhagen, v.28, n.12, p.1137-1144, Dec. 2001.

DINARELLO, C.A. Biologic basis for Interleukin- 1 in disease. Blood, Washington, DC, v.87, n.6, p.2095-2147, M ar. 1996.

DINARELLO, C.A.; FANTUZZI, G. Interleukin- 18 and host defense against infection. J. Infect. D is., Chicago, v.187, p.370-384, June 2003. Suppl. 2.

DUFF, G.W. M olecular genetics of cytokines: cytokines in chronic inflammatory disease. In: TH OMPSON, A. The cytokine handbook. London: Academic Press, 1998. p.21-33.

FOLWACZNY, M. et al. Polymorphisms of the interleukin-18 gene in periodontitis patients. J. Clin. Periodontol., Copenhagen, v.32, n.5, p.530-534, M ay 2005a.

FOLWACZNY, M. et al. Prevalence of the 295 T-to-C promoter polymorphism of the interleukin (IL)-16 gene in periodontitis. Clin.

Exp. Immunol., Oxford, v.142, n.1, p.188192, 0 ct. 2005b.

FUJIHASH I, K. et al. Cytokineand periodontal disease: immunopathological role of interleukins for B cell response in chronic inflamed gingival tissues. J. Periodontol., C hicago, v.64, p.400-406, M ay 1993. Suppl. 5.

GERDES, N. et al. Expression of interleukin (IL)- 18 and functional II-18 receptor on human vascular endothelial cells, smooth muscle cells and macrophagis implications for atherogenesis. J. Exp. Med., New York, v.195, n.2, p.245257, Jan. 2002.

GONZALES, J.R. et al. Analysis of genetic polymorphisms at the interleukin-10 loci in aggressive and chronic periodontitis. J. Clin. Periodontol., Copenhagen, v.29, n.9, p.816822, Sept. 2002.

GONZALES, J.R. et al. Comparison of interleukin-1 genotypes in two populations with aggressive periodontitis. Eur. J. 0 ral Sci., Copenhagen, v.111, n.5, p.395-399, 0 ct. 2003.
GONZALES, J.R. et al. Interleukin-4 gene polymorphisms in Japanese and caucasian patients with aggressive periodontitis. J. Clin. Periodontol., Copenhagen, v.31, n.5, p.384389, M ay 2004.

GORE, E.A. et al. Interleukin-1beta +3953 allele 2: association with disease status in adult periodontitis. J. Clin. Periodontol., Copenhagen, v.25, n.10, p.781-785, O ct. 1998.

GREENSTEIN, G.; HART, T.C. A critical assessment of interleukin - 1 (IL-1) genotyping when used in a genetic susceptibility test for severe chronic periodontitis. J. Periodontol., Chicago, v.73, n.2, p.231-247, Feb. 2002.

GUZMAN, S. et al. Association between interleukin-1 genotype and periodontal disease in a diabetic population. J. Periodontol., Chicago, v.74, n.8, p.1183-1190, Aug. 2003.

HAJEER, A.H . et al. IL-10 gene promoter polymorphisms in rheumatoid arthritis. Scand. J. Rheumatol., Stockholm, v.27, n.2, p.142145, 1998.

HART, T.C. Genetic considerations of risk in human periodontal disease. Curr. 0 pin. Periodontol., Philadelphia, p.3-11, 1994.

H ART, T.C. Genetic risk factors for early onset periodontitis. J. Periodontol., Chicago, v.67, p.355-366, 1996.

HART, T.C.; KORNMAN, K.S. Genetic factors in the pathogenesis of periodontitis.

Periodontol. 2000, Copenhagen, v.14, p.202215, June 1997.

HASSELL, T.M.; H arris, E.L. Genetic influences in caries and periodontal disease.

Crit. Rev. 0 ral Biol. M ed., Alexandria, v.6, n.4, p.319-342, 1995.

HOLLA, L.I. et al. Analysis of the interleukin6 gene promoter polymorphisms in Czech patients with chronic periodontitis. J. Periodontol., Chicago, v.75, n.1, p.30-36, Jan. 2004.

MICHEL, J. et al. Interleukin- 4 polymorfisms in early onset periodontitis. J. Clin. 
Periodontol., Copenhagen, v.28, n.5, p.483, M ay 2001.

JANEWAY, C.A. et al. Imunologia: o sistema imunológico na saúde e na doença. 4.ed. Porto Alegre: Artes M édicas Sul, 2000.

KANG, B.Y. et al. Two polymorphisms of interleukin-4 genein Korean adult periodontitis. Arch. Pharm. Res., Seoul, v.26, n.6, p.482486, June 2003.

KIN ANE, D.F.; HART, T.C. Genes and gene polymorphisms associated with periodontal disease. C rit. Rev. 0 ral Biol. M ed., Alexandria, v.14, n.6, p.430-449, 2003.

KORNMAN, K.S. et al. The interleukin-1 genotype as a severity factor in adult periodontal disease. J. Clin. Periodontol., Copenhagen, v.24, n.1, p.72-77, Jan. 1997.

LABERGE, $S$. et al. Increased expression of interleukin-16 in bronchial mucosa of subjects with atopic asthma. Am. J. Respir. Cell Mol. Biol., New York, v.17, n.2, p.193-202, Aug.1997.

LALANI, I.; BHOL, K.; AHMED, A.R. Interleukin-10: biology, role in inflammation and autoimmunity. Ann. Allergy Asthma Immunol., M CLean, v.79, p.469-484, 1997.

LANDI, L. et al. Host mechanisms in the pathogenesis of periodontal disease. Curr. 0 pin. Periodontol., Philadel phia, v.4, p.3-10, 1997.

LAN G, N.P. et al. Effect of interleukin -1 polymorphisms on gingival inflamation assessed by bleeding on probing in a periodontal maintenance population. J. Periodont. Res., Copenhagen, v.35, p.102-107, A pr.2000.

M AT HY, N.L. et al. Interleukin-16 stimulates the expression and production of pro- inflammatory cytokines by human monocytes. Immunology, 0 xford, v.100, n.1, p.63-69, M ay 2000.

MEISEL, P. et al. The interleukin-1 polymorphism, smoking, and the risk of periodontal disease in the population-based
SH IP study. J. D ent. Res., Alexandria, v.82, n.3, p.189-193, M ar. 2003.

MICH ALOWICZ, B.S. Genetic and heritable risk factors in periodontal disease. J. Periodontol., Chicago, v.65, p.479-488, M ay 1994. Suppl. 5.

MICHALOWICZ, B.S. et al.Evidence of a substantial genetic basis for risk of adult periodontitis. J. Periodontol., Chicago, v.71, n.11, p.1699-1707, N ov. 2000.

M OREIRA, P.R. et al. A functional interleukin1 beta gene polymorphism is associated with chronic periodontitis in a sample of Brazilian individuals. J. Periodont. Res., Copenhagen, v.40, n.4, p.306-311, Aug. 2005.

NARES, S. The genetic relationship to periodontal disease. Periodontol. 2000, Copenhagen, v.32, p.36-49, 2003.

PAPAPANOU, P.N . et al. Interleukin-1 gene polymorphism and periodontal status: a casecontrol study. J. Clin. Periodontol., Copenhagen, v.28, n.5, p.389-396, M ay 2001.

PARKHILL, J.M. et al. Association of interleukin-1 gene polymorphisms with earlyonset periodontitis. J. Clin. Periodontol., Copenhagen, v.27, n.9, p.682-689, Sept. 2000.

PAUL, W.E. Interleukin-4 : a prototypic immunoregulatory lymphokine. Blood, Washington, DC, v.77, n.9, p.1859-1870, M ay 1991.

PONTES, C.C. et al. Interleukin-4 gene polymorphism and its relation to periodontal disease in a Brazilian population of African heritage. J. D ent., Kidlington, v.32, n.3, p.241-246, M ar. 2004.

SCAREL-CAM IN AGA, R.M . et al. Interleukin 10 gene promoter polymorphisms are associated with chronic periodontitis. J. Clin. Periodontol., Copenhagen, v.31, n.6, p.443448, June 2004.

SCAREL-CAMINAGA， R.M. et al. Investigation of an IL-2 polymorphism in patients with different levels of chronic 
periodontitis. J. Clin. Periodontol., Copenhagen, v.29, n.7, p.587-591, July 2002.

SCHREIBER, $S$. Monocytes or $T$ cells in Crohn's disease: does II-16 allow both to play at that game? Gut, London, v.49, p.747-748, 2001.

SO GA, Y. et al. Tumor necrosis factor-alpha gene (TN F-alpha) -1031/-863, -857 singlenucleotide polymorphisms (SNPS) are associated with severe adult periodontitis in Japanese. J. Clin. Periodontol., Copenhagen, v.30, n.6, p.524-531, June 2003.

TAI, H. et al. Association of interleukin-1 receptor antagonist gene polymorphisms with early onset periodontitis in Japanese. J. C Clin. Periodontol., Copenhagen, v.29, n.10, p.882888, 0 ct. 2002.
TE VELDE, A.A. et al. Interleukin -4 inhibit secretion of IL-1b tumor necrosis factor-a and IL- 6 by human monocytes. Blood, Washington, DC, v.76, n.7, p.1392-1397, O ct. 1990. TREVILATTO, P.C. et al. Polymorphism at position -174 of IL- 6 gene is associated with susceptibility to chronic periodontitis in a caucasian Brazilian population. J. Clin. Periodontol., Copenhagen, v.30, n.5, p.438442, M ay 2003.

YAMAZAKI, K. et al. Interleukin-10 gene promoter polymorphism in Japanese patients with adult and early-onset periodontitis. J. Clin. Periodontol., Copenhagen, v.28, n.9, p.828-832, Sept. 2001.

Recebido em / Received: 01/06/2006 Aceito em / Accepted: 25/08/2006 